\title{
The Role of Pro-Adrenomedullin as a Marker of Severe Bacterial Infection in Children: A Review
}

\author{
Silvia Garazzino *(D), Elena Altieri and Marco Denina \\ Paediatric Infectious Diseases Unit, University of Turin, Regina Margherita Children's Hospital, \\ Piazza Polonia 94, 10126 Turin, Italy \\ * Correspondence: silvia.garazzino@unito.it; Tel.: +39-0113131704; Fax: +39-0113135550
}

Received: 30 April 2019; Accepted: 4 July 2019; Published: 8 July 2019

\begin{abstract}
Sepsis is still related to high mortality and hospitalization rates in the paediatric population despite medical progress. Prompt recognition of sepsis and consequent initiation of an appropriate treatment are essential elements in securing a positive outcome in adults and children. In such scenario, various biomarkers have been studied to support the clinician in the diagnosis of severe infections, in the identification of patients at risk of progression towards sepsis as well as in the evaluation of the response to treatment. In children, the performance of C-reactive protein and procalcitonin has been largely investigated, whereas data on newer biomarkers of sepsis are scant. The aim of the present review is to summarise the available data on the use of pro-adrenomedullin in the paediatric age.
\end{abstract}

Keywords: adrenomedullin; children; neonates; sepsis; severe infections

\section{Introduction}

Serious bacterial infection (SBI) is a major cause of morbidity and mortality in children worldwide and it is responsible for about $60 \%$ of childhood mortality [1]. Early recognition of sepsis and the prompt administration of appropriate antibiotic treatment are crucial elements for preventing the evolution of sepsis towards septic shock. Various diagnostic markers of sepsis have been suggested in a variety of clinical settings and age groups in order to facilitate early diagnosis of SBI and to predict prognosis [2].

In recent years, the interest of the scientific community has focused on adrenomedullin (ADM), a peptide that is produced by heart, adrenal medulla, lungs, kidneys, and vascular endothelium during physiological stress. ADM was initially studied for its vasodilating properties; more recently, a central role of ADM in the inflammatory response has been demonstrated. Lipopolysaccharides and pro-inflammatory cytokines, such as TNF- $\alpha$ and IL- $1 \beta$, rapidly promote ADM production through increased ADM gene expression in several tissues [3]. The release of ADM into the bloodstream regulates the vascular tone, guaranteeing adequate organ perfusion; it also results in potent pore-mediated antibacterial activity and in immunomodulation through the induction of apoptosis.

The ADM gene is located at the short arm of human chromosome 11 (p11.1-3) and it consists in four exons and three introns. The mRNA encodes the information for the synthesis of a preprohormone of 185 aminoacids, known as preproadrenomedullin, which is subsequently degraded into a 164-aminoacid-peptide, called proadrenomedullin (ProADM), through cleavage of the signal peptide [3]. ProADM is utterly cleaved by an endogenous peptidase, peptidylhydroxyglycine $\alpha$-amidating lyase, into four different peptides: ADM, the aminoterminal peptide of ProADM (PAMP), adrenotensin, and midregional proadrenomedullin (MR-ProADM) [4]. ProADM and ADM are secreted in equimolar amounts during this post-translational modulation. However, ADM is rapidly metabolized and 
eliminated from the bloodstream, which makes its levels hardly measurable. ProADM displays higher stability in circulation and it is easily detectable.

In the adult population, it has been documented that ADM and ProADM levels rapidly rise in sepsis and serious bacterial infections, that they are related to the severity of the disease, and that they accurately predict the risk of organ failure and mortality.

Despite that literature being rich of studies on ProADM in the adult population, data on its performance in the paediatric setting are limited.

The purpose of this review is to summarise the available data on when and how ProADM dosing is useful in paediatric ages.

\section{Materials and Methods}

We conducted a literature review to investigate the role of ProADM in children, while applying the methodology described below.

An electronic PubMed search was performed while using the following key words: (proadrenomedullin[Title/abstract]) AND (children[Title/abstract] OR newborn[Title/abstract] OR infants[Title/abstract]OR neonates[Title/abstract]). A total of 30 articles were found. Reviews, opinion papers, and articles not related to infectious diseases were excluded. The full text of the remaining studies was then evaluated, while considering the following items: authors, country and year of publication, characteristics of the population analysed, performance of ProADM, and eventual comparison with other biomarkers or scoring systems.

A total of 16 studies were finally selected (Table 1). Most of the studies had a low level of evidence (IV to VI) being case-control or cohort studies.

As the study was designed as a narrative literature review, no ethical committee permission was deemed to be necessary.

Table 1. Summary of studies on pro-adrenomedullin (ProADM) in the paediatric population.

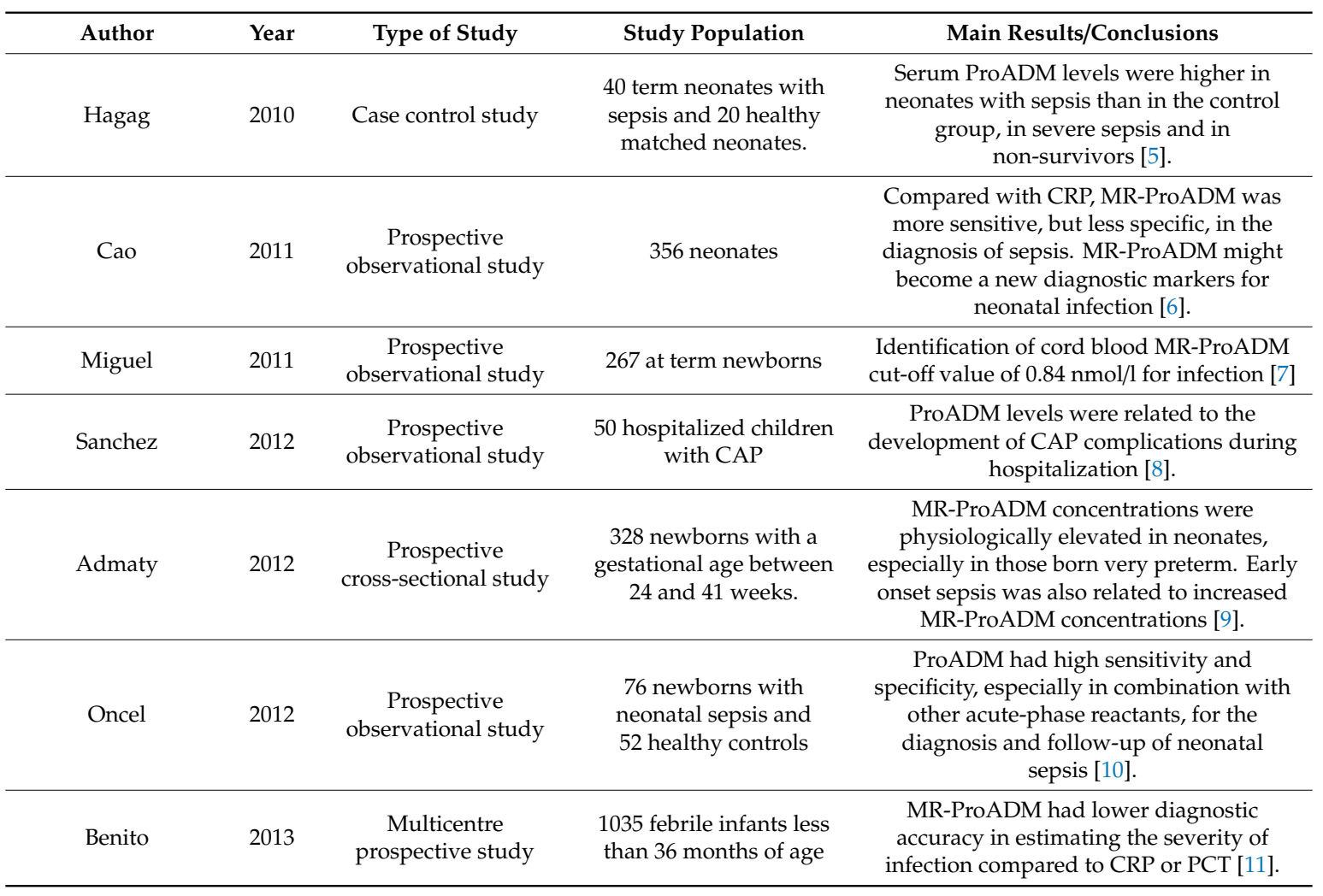


Table 1. Cont.

\begin{tabular}{|c|c|c|c|c|}
\hline Author & Year & Type of Study & Study Population & Main Results/Conclusions \\
\hline Jordan & 2014 & $\begin{array}{c}\text { Prospective } \\
\text { observational study }\end{array}$ & 95 septic children & $\begin{array}{l}\text { MR-ProADM levels were a good indicator } \\
\text { of disease severity and had a better } \\
\text { reliability than PCT and CRP for predicting } \\
\text { in-hospital mortality [13]. }\end{array}$ \\
\hline Alcoba & 2015 & $\begin{array}{c}\text { Prospective cohort } \\
\text { study }\end{array}$ & $\begin{array}{l}88 \text { children aged } 0 \text { to } \\
16 \text { years presenting to } \\
\text { the paediatric emergency } \\
\text { with CAP }\end{array}$ & $\begin{array}{l}\text { ProADM seemed a reliable predictor for } \\
\text { complicated CAP, but was similar to CRP in } \\
\text { sensitivity and specificity [14]. }\end{array}$ \\
\hline Mìguez & 2016 & $\begin{array}{c}\text { Single-centre } \\
\text { prospective } \\
\text { observational study }\end{array}$ & $\begin{array}{l}136 \text { children with } \\
\text { suspected acute } \\
\text { appendicitis }\end{array}$ & $\begin{array}{l}\text { MR-ProADM was not helpful in the early } \\
\text { diagnosis of AA. The combination of low } \\
\text { CRP and low MR-ProADM levels was } \\
\text { useful for the identification of children with } \\
\text { a low risk of AA [15]. }\end{array}$ \\
\hline Esposito & 2016 & $\begin{array}{c}\text { Prospective } \\
\text { multicentre study }\end{array}$ & $\begin{array}{l}433 \text { otherwise healthy } \\
\text { children hospitalized for } \\
\text { radiologically } \\
\text { confirmed CAP }\end{array}$ & $\begin{array}{l}\text { In children with CAP, MR-ProADM blood } \\
\text { levels poorly differentiated bacterial from } \\
\text { viral diseases or severe from mild cases [16]. }\end{array}$ \\
\hline Oikonomopoulou & 2018 & $\begin{array}{l}\text { Prospective, } \\
\text { observational, } \\
\text { multicentre study } \\
\text { conducted in } \\
6 \text { paediatric emergency } \\
\text { departments. }\end{array}$ & $\begin{array}{l}285 \text { children with } \\
\text { suspected AA }\end{array}$ & $\begin{array}{l}\text { Children with AA, especially if } \\
\text { complicated, had higher ProADM values } \\
\text { than children with non infectious } \\
\text { abdominal pain. The combination of low } \\
\text { values of ProADM and CRP was useful to } \\
\text { select children with low risk of AA [17]. }\end{array}$ \\
\hline Fahmey & 2018 & Prospective study & $\begin{array}{l}60 \text { newborns with } \\
\text { proven sepsis and } \\
30 \text { healthy neonates. }\end{array}$ & $\begin{array}{l}\text { ProADM was a reliable biomarker for } \\
\text { neonatal sepsis. High ProADM levels were } \\
\text { associated with mortality and were an early } \\
\text { indicator of disease outcome [20] }\end{array}$ \\
\hline
\end{tabular}

CRP: C-reactive protein; PCT: procalcitonin; CAP: community acquired pneumonia; AA: acute appendicitis.

\subsection{Proadrenomedullin in Children with Serious Bacterial Infections}

The utility of ProADM in children with SBIs is still debated.

A multicentre study investigated 1035 well appearing febrile infants that were seen in a Spanish paediatric emergency department and compared MR-ProADM levels with C-reactive protein (CRP) and procalcitonin (PCT) in diagnosing SBIs. The plasma levels of MR-ProADM were significantly higher in patients with invasive bacterial infections (such as sepsis or meningitis) than in children with localized infections (i.e., urinary tract infections-UTIs—or pneumonia), but had a lower diagnostic accuracy in estimating the severity of infection when compared to CRP or PCT. The amount of septic children was limited (only 16 patients). According to authors' conclusions, ProADM alone is useless in identifying SBIs, but can be helpful in combination with other markers. Noteworthy, the threshold value for MR-ProADM for bacterial infection was set at $0.75 \mathrm{nmol} / \mathrm{L}$. In the adult population, MR-ProADM cut-offs for sepsis and severe sepsis have been settled at 1.8 and $2.5 \mathrm{nmol} / \mathrm{L}$, respectively. This difference may have influenced the interpretation of the results [11].

In a following study, MR-ProADM resulted a good indicator of disease severity in critically ill children with sepsis and had better accuracy than PCT and CRP in predicting in-hospital mortality 
(AUC was 0.77 for MR-ProADM, 0.67 for PCT and 0.63 for PCR). When compared to the PRISM III score, a MR-ProADM cut off of $2.2 \mathrm{nmol} / \mathrm{L}$ had similar accuracy [13]. Similar conclusions are reported in another prospective observational study that was performed by Rey et colleagues: in a population of 254 children that were admitted to a paediatric intensive care unit, MR-ProADM had $93 \%$ sensitivity and $76 \%$ specificity to differentiate the high mortality risk group, with an AUC of 0.87 . MR-proADM levels $>0.80 \mathrm{nmol} / \mathrm{L}$ appeared to be useful in identifying children at elevated risk of death [12].

\subsection{Neonatal Sepsis}

Sepsis is one of the major causes of death during the neonatal period. Mortality and comorbidities remain high despite recent advances in treatment. Early diagnosis represents an important challenge for neonatologists, because clinical signs are difficult to differentiate from other non-infective neonatal conditions. A reliable and rapid diagnostic marker is needed to guide the initiation of antimicrobial agents.

In neonates, cut-offs for ProADM are currently under evaluation.

In 2010, Míguel et colleagues measured MR-ProADM in the cord blood of 194 healthy newborns without risk factors for sepsis and in 73 newborns with risk factors for sepsis at delivery. They finally identified a reference cut-off of MR-ProADM of $0.84 \mathrm{nMol} / \mathrm{L}(0.5-1.38)$ for healthy newborns [7].

In 2012, Oncel and colleagues measured the plasmatic levels of ProADM in three groups of patients: newborns with proven sepsis (31 patients), with clinical sepsis (45 patients), and healthy controls (52 patients). They found that the ProADM levels were significantly higher in newborns with sepsis as compared with healthy controls, and also that ProADM more rapidly decreased, in patients with controlled sepsis, as compared to CRP. They identified a cut-off value of $3.9 \mathrm{nMol} / \mathrm{L}$ for ProADM to discriminate proven or clinical sepsis from non-septic conditions, with an AUC, respectively, of 0.97 and 0.98. In this study, ProADM appeared to be useful for the diagnosis and follow-up of early onset sepsis (EOS) in neonates, but the concomitant use with conventional acute-phase reactants was suggested [10].

A cross-sectional prospective study, which was conducted between March 2009 and December 2010 in Switzerland, evaluated MR-ProADM plasma levels in 328 infants that were born between 24 and 41 weeks gestational age (GA). The lower the GA, the higher MR- ProADM plasma concentrations were at birth and at 2-3 days of life. Irrespective of GA and birth weight (BW), MR-ProADM plasma cord blood concentrations at birth were increased in infants with EOS, signs of antenatal cardiovascular compromise, and born from mothers with chorioamnionitis. Finally, the need for respiratory support was associated with higher MR-ProADM plasma concentrations at 2-3 days of life [9].

The efficacy of ProADM in detecting neonatal sepsis was demonstrated in several other studies. ProADM levels were significantly higher in the sepsis group than in the control group $(p<0.001)$ in a prospective study on 90 neonates ( 30 healthy and 60 with microbiologically documented sepsis). In this study, $4.3 \mathrm{nmol} / \mathrm{L}$ was identified as the optimal cut-off for ProADM: this value had a sensitivity of $93.3 \%$ and a specificity of $86.7 \%$. The ProADM levels were also significantly higher in non-survivors $(p=0.001)[20]$.

Similarly, Kannan et al. found higher ProADM levels in septic neonates when compared to controls (median 31.8 vs $5.1 \mathrm{pMol} / \mathrm{mL}$ ). 90\% of neonates with increased ProADM died and $92.5 \%$ of neonates with decreased ProADM survived. The serial monitoring of ProADM levels had a significant role in predicting prognosis: indeed, a decrease in ProADM level by $10 \mathrm{pMol} / \mathrm{mL}$ was associated with a $99 \%$ probability of survival [19].

The role of ProADM as predictor of outcome in sepsis was also documented in a case control study on 40 newborns with sepsis that were admitted to neonatal intensive care unit (NICU) when compared with 20 healthy neonates of matched GA and BW. A significant increase in the ProADM plasma levels was found in septic patients, as compared with controls, but also in patients with sepsis who did not survive as compared with survivors [5]. 
A wider population of neonates $(n=356)$ was studied by Cao et al. The serum levels of MR-ProADM were significantly increased in both neonatal infection groups (severe and mild), and it was higher in the severe infection group. Moreover, the serum MR-ProADM levels were higher in neonates with culture-proven sepsis than in those with clinical sepsis. When compared with CRP (cut-off level: $8 \mathrm{mg} / \mathrm{L}$ ), MR-ProADM cut-off of $1.12 \mathrm{nMol} / \mathrm{L}$ was more sensitive, but less specific, in identifying neonatal sepsis [6].

\subsection{Pneumonia}

Community-acquired-pneumonia (CAP) is the leading cause of child morbility worldwide. The decision to hospitalize the patient and to start an intravenous antimicrobial treatment mainly depends on the severity of the disease and on the risk of complications, such as sepsis, empyema, or abscess. Several attempts to find an effective biomarker to identify and to stratify bacterial CAP in children have been made in recent years, but only few studies analysed the role of ProADM, and their results are sometimes contradictory.

A prospective observational study, including hospitalized children with CAP, showed that ProADM was higher in complicated pneumonia (2.3190 vs. $1.1758, p=0.013)$ : in particular, in children with pleural effusion median ProADM was $2.9440 \mathrm{nmol} / \mathrm{L}$ as compared to $1.1373 \mathrm{nMol} / \mathrm{L}$ in those without effusion $(p<0.001)$. On the contrary, the CRP levels did not differ between patients with complicated and uncomplicated CAP (206 vs. $254.5 \mathrm{mg} / \mathrm{L}, p=0.558$ ). The ProADM levels were not related to other variables, such as oxygen therapy, duration of fever, length of hospital stays, duration of parenteral antibiotic therapy, admission to ICU, and unilobar or multilobar CAP at admission [8].

A similar conclusion was drawn by another study on children up to 16 years of age with CAP, where a statistically significant increase of ProADM levels was associated with bacteraemia or empyema (at logistic regression $p=0.007$ and 0.036, respectively). A significant difference in ProADM median values was again found in complicated cases $(0.18$ vs. $0.08 \mathrm{nmol} / \mathrm{L}, p=0.039)$ and in bacteraemic CAP ( 0.40 vs. $0.08 \mathrm{nmol} / \mathrm{L}, p=0.02)$. A cut off of ProADM $>0,16 \mathrm{nmol} / \mathrm{L}$ had $100 \%$ sensitivity $(95 \% \mathrm{CI}$ $39.8-100.0)$ and $70 \%$ specificity (95\% CI $58.7-79.7)$ for bacteraemia and a cut off of $0.16 \mathrm{nmol} / \mathrm{L}$ was as accurate as CRP $<100 \mathrm{mg} / \mathrm{L}$ and better than band neutrophils $(<1.5 \mathrm{G} / \mathrm{L})$ or leucocytosis $(<15 \mathrm{WBC} / \mathrm{L})$ to rule out bacteraemia (sensitivity and NPV $=10 \%$ ) [14].

In contrast, in a recent prospective, multicentre study, neither soluble triggering receptor expressed on myeloid cells-1 (sTREM-1), midregional proatrial natriuretic peptide (MR-proANP), nor MR-ProADM were able to differentiate bacterial from viral CAP or to identify severe cases. However, the levels of MR-ProADM that were found in bacterial pneumonia were significantly higher than those in undetermined CAP cases $(0.50 \pm 0.94$ vs 0.35 vs $\pm 0.17, p<0.05)$ [16].

Finally, ProADM appeared to be the best biomarker for severity stratification of CAP as compared to CRP and IL-1 $\beta$ levels. ProADM logistic regression analysis revealed that the respiratory clinical score $>6$ points and ProADM values $>1.75 \mathrm{nmol} / \mathrm{L}$ combination had the most accurate results in predicting the development of complications in CAP (OR: 15.38, 95\% CI 1.35-166.66, $p=0.027$ ) [18].

\subsection{Acute Abdominal Pain}

Acute appendicitis (AA) is a frequent surgical disease in the paediatric population. In at least one-third of cases, the clinical course is atypical, making the diagnosis of AA sometimes challenging. The timing of identification of AA is crucial in preventing progression toward perforation, while excluding AA allows for avoiding unnecessary appendectomies. Several diagnostic tools have been developed to facilitate AA identification, such as laboratory tests, clinical assessment scales, and imaging tests. However, only two studies analysed the role of ProADM in pediatric AA.

In a prospective analytical-observational, multicentre study mean ProADM concentrations were significantly higher $(p<0.001)$ in children with AA $(0.51 \mathrm{nMol} / \mathrm{L} ; \mathrm{SD} 0.16)$ as compared to children with abdominal pain due to non-infectious causes $(0.44 \mathrm{nMol} / \mathrm{L} ; \mathrm{SD} 0.14)$ and in patients with complicated AA $(p=0.005,0.64 \mathrm{nmol} / \mathrm{L} ; \mathrm{SD} 0.17)$ as compared to non-complicated cases $(0.50 \mathrm{nmol} / \mathrm{L} ; \mathrm{SD} 0.15)$. 
However, ProADM alone resulted inadequate in the early identification of AA. The combination of low MR-proADM values $(\leq 0.35 \mathrm{nmol} / \mathrm{L})$ and low CRP values $(\leq 1.25 \mathrm{mg} / \mathrm{dL})$ was indeed useful for stratifying children with abdominal pain at low risk of appendicitis, with a predictive value of $93 \%$ [17].

A second prospective study, which was conducted in children who presented to the emergency department with suspected AA, found a mean concentration of MR-ProADM that was significantly higher in children with AA than in children with non-specific abdominal pain (NAP). Similar to the previous study, the performance of MR-ProADM alone was not optimal; however, if combined with a CRP level of $<0.3 \mathrm{mg} / \mathrm{dl}$, a cut-off for ProADM of $<0.34 \mathrm{nmol} / \mathrm{L}$ had a $100 \%$ negative predictive value [15].

\subsection{Urinary Tract Infection}

UTI is another common disease in children. A delay in diagnosis and treatment of complicated UTI with pyelonephritis is an independent risk factor for developing scars and renal insufficiency. The performance of ADM has mostly been studied in glomerular diseases in adults. In children, four studies are available on the performance ADM in urinary tract infections. It seems that ADM is significantly higher in patients with UTIs despite the small sample size of such studies, especially if with pyelonephritis, than in the controls [21-23].

Conversely, no studies on ProADM in children with UTIs were found in the literature.

\subsection{Neutropenic Fever in Oncologic Children}

In childhood cancers, the severe and prolonged neutropenia that follows chemotherapy, results in a high risk of severe infections. In neutropenic febrile children, infection needs to be ruled out and differentiated from febrile reactions that are induced by chemotherapeutic agents and blood products. Different biomarkers have been investigated to identify those patients who need antimicrobial treatment; however, only two studies evaluated the role of ADM. In this setting, the ADM levels accurately distinguished infection from inflammation, predicting the presence of culture positivity and identifying high-risk patients [24,25]. However, the ADM levels poorly correlated with the severity of infection.

To date, no studies on ProADM in neutropenic febrile children are available in the literature.

\section{Discussion}

Our literature search only found 16 studies that were focused on the use of ProADM in the pediatric setting. Such studies are quite heterogeneous, both in terms of the population enrolled and the type of infection investigated.

Most studies measured MR- or ProADM on plasma samples; a few studies directly measured the serum levels, while a bunch did not specify the type of sample collected. Therefore, both plasma and serum appear to be suitable materials for MR-/ProADM dosing.

Different dosing techniques were used for Pro-ADM and MR-ProADM measurement and that might have affected study results. The most frequently used technique was an automated immunofluorescent assay by the Kryptor Analyzer (Brahms Diagnostica, Hennigsdorf, Germany). A few authors used a quantitative sandwich enzyme immuno-assay technique that was produced in Wuhan, China. One study used a commercial radioimmunoassay kit (Cusabio Biotech, Wilmington, Germany) and the assay technique was not specified in one study.

From a general point of view, the performance of ProADM in neonates and children was satisfactory overall. Analysing specific situations, it seems that ProADM alone may be a good indicator of intra-hospital mortality in critically ill children, especially if it is tested in the first $24 \mathrm{~h}$ from ICU admission and if a high cut-off value $(>2.2 \mathrm{nmol} / \mathrm{L})$ is used. However, its capacity of identifying invasive bacterial infections in well appearing febrile infants is higher if ProADM is used in combination with other biomarkers, such as PCT. No cut-off value has been established for the latter situation. When 
compared to the PRISM III score, MR-ProADM (cut off of $2.2 \mathrm{nmol} / \mathrm{L}$ ) has similar accuracy, but is undoubtfully easier to measure (calculating PRISM III score requires the determination of 14 variables).

The mortality predictive value of $2.2 \mathrm{nmol} / \mathrm{L}$ in paediatric sepsis is not far from what is found in adults. In the TRIAGE study (a multinational, prospective, observational cohort study, including 7132 adults admitted for a medical urgency) copeptin, PCT and Pro-ADM strongly predicted the risk of death, ICU admission, and high initial triage priority. For mortality prediction, ProADM had the best discrimination value, with an AUC of 0.83 when compared to 0.78 for copeptin and 0.75 for PCT. The median level of ProADM in non-survivor was $1.8 \mathrm{nmol} / \mathrm{L}$ [26].

ProADM seems to also be useful for an early diagnosis of neonatal EOS (especially in combinations with other marker of sepsis, such as PCR and PCT) and for predicting response to antimicrobial therapy. In neonates, especially if preterm, levels of ProADM are physiologically elevated, requiring cut-off values for infection that are higher than those set in older children. Two different cut-offs $(3.9 \mathrm{nmol} / \mathrm{L}$ and $4.3 \mathrm{nmol} / \mathrm{L}$ ) $[10,20]$ were significantly related to EOS, and, similarly to PCT, the ProADM trend in the first three days of life was a good predictor of neonatal mortality.

In pneumonia, ProADM can be useful for identifying complicated CAP, especially those with pleural effusion. Similarly, in adults, a recent systematic review and meta-analysis, including a total of 4119 patients that were admitted to the emergency department, demonstrated that MR-ProADM is predictive of increased complications (overall area under the SROC curve 0.74 ) and higher mortality rates (RR 6.16, 95\% CI 4.71-8.06) in patients with CAP [27]. Another meta-analysis, including 10,319 adult patients with CAP, showed that ProADM was superior to PCT and CRP in predicting short-term mortality in CAP [28].

Finally, the combination of low ProADM and CRP values may help to identify the children with abdominal pain with a low risk of appendicitis who do not benefit from antibiotic treatment and that may be safely discharged from hospital.

No studies on ProADM in febrile neutropenic children or in children with UTIs were found, even if in this setting ADM has demonstrated some utility. In the adult population, a secondary analysis of a randomized, placebo-controlled trial involving patients with febrile UTI, MR-ProADM levels at admission were positively correlated with length of hospital stay $(p<0.001)$, bacteraemia $(p<0.001)$, initial intravenous treatment $(p<0.001)$, and time to defervescence $(p<0.001)$ [29]. In a study that included 340 febrile adult patients with hematologic malignancies, only ProADM, and not PCT, was significantly higher in patients with localized bacterial infections, when compared to those without infection $(p=0.019)$ and in patients with definite sepsis than those with systemic inflammatory response syndrome (SIRS) $(p=0.023)$. ProADM levels significantly decreased in response to appropriate antimicrobial therapy [30].

\section{Conclusions}

In conclusion, ProADM seems to be useful in the paediatric population to identify children with severe infections, especially neonates, but at present clinical data are still limited and they suggest that ProADM should be used in combination with other biomarkers, rather than as a unique tool. However, the number of papers available in literature is limited to date: further research in this field is necessary in order to improve the early detection of sepsis in children. Future perspectives include the potential use of ProADM to rule out other non-infectious diseases that are typically encountered in children that may mimic sepsis, such as Kawasaki Disease or systemic juvenile arthritis.

Author Contributions: Each author made substantial contributions to the conception and design of the work, the acquisition, analysis and interpretation of data, drafted the work and substantively revised and approved the submitted version; each author agrees to be personally accountable for the author's own contributions and for ensuring that questions related to the accuracy or integrity of any part of the work are appropriately investigated, resolved, and documented in the literature.

Acknowledgments: The authors disclose no funding of the study.

Conflicts of Interest: The authors declare no conflict of interest. 


\section{References}

1. Weiss, S.L.; Fitzgerald, J.C.; Pappachan, J.; Wheeler, D.; Jaramillo-Bustamante, J.C.; Salloo, A.; Singhi, S.C.; Erickson, S.; Roy, J.A.; Bush, J.L.; et al. Global Epidemiology of Pediatric Severe Sepsis: The Sepsis Prevalence, Outcomes, and Therapies Study. Am. J. Respir. Crit. Care Med. 2015, 191, 1147-1157. [CrossRef] [PubMed]

2. Lanziotti, V.S.; Póvoa, P.; Soares, M.; Silva, J.R.L.; Barbosa, A.P.; Salluh, J.I.F. Use of biomarkers in pediatric sepsis: Literature review. Rev. Bras. Ter. Intensiva 2016, 28, 472-482. [CrossRef] [PubMed]

3. Zudaire, E.; Portal-Núñez, S.; Cuttitta, F. The central role of adrenomedullin in host defense. J. Leukoc. Boil. 2006, 80, 237-244. [CrossRef] [PubMed]

4. Valenzuela-Sánchez, F.; Valenzuela-Méndez, B.; Rodríguez-Gutiérrez, J.F.; Estella-García, Á.; González-García, M.Á. New role of biomarkers: Mid-regional pro-adrenomedullin, the biomarker of organ failure. Ann. Transl. Med. 2016, 4, 329. [CrossRef] [PubMed]

5. Hagag, A.A.; Elmahdy, H.S.; Ezzat, A.A. Prognostic value of plasma pro-adrenomedullin and antithrombin levels in neonatal sepsis. Indian Pediatr. 2011, 48, 471-473. [CrossRef] [PubMed]

6. Cao, Y.; Xia, Q.; Chen, C.; Yang, Y. Precursors of adrenomedullin, endothelin and atrial natriuretic peptide as diagnostic markers of neonatal infection. Acta Paediatr. 2012, 101, 242-246. [CrossRef]

7. Miguel, D.; Prieto, B.; Costa, M.; Coto, D.; Álvarez, F.V. Cord blood plasma reference intervals for potential sepsis markers: Pro-adrenomedullin, pro-endothelin, and pro-atrial natriuretic peptide. Clin. Biochem. 2011, 44, 337-341. [CrossRef]

8. Sardà Sánchez, M.; Hernández, J.C.; Hernández-Bou, S.; Teruel, G.C.; Rodríguez, J.V.; Cubells, C.L. Pro-adrenomedullin usefulness in the management of children with community-acquired pneumonia, a preliminar prospective observational study. BMC Res. Notes 2012, 5, 363. [CrossRef]

9. Admaty, D.; Benzing, J.; Burkhardt, T.; Lapaire, O.; Hegi, L.; Szinnai, G.; Morgenthaler, N.G.; Bucher, H.U.; Bührer, C.; Wellmann, S. Plasma midregional proadrenomedullin in newborn infants: Impact of prematurity and perinatal infection. Pediatr. Res. 2012, 72,70-76. [CrossRef]

10. Oncel, M.Y.; Dilmen, U.; Erdeve, O.; Ozdemir, R.; Calisici, E.; Yurttutan, S.; Canpolat, F.E.; Oguz, S.S.; Uras, N.; Çalışıc1, E.; et al. Proadrenomedullin as a prognostic marker in neonatal sepsis. Pediatr. Res. 2012, 72, 507-512. [CrossRef]

11. Benito, J.; Luaces-Cubells, C.; Mintegi, S.; Astobiza, E.; Martinez-Indart, L.; Valls-Lafont, A.; Garcia-Garcia, J.J. Lack of value of midregional pro-adrenomedullin and C-terminal pro-endothelin-1 for prediction of severe bacterial infections in infants with fever without a source. Eur. J. Pediatr. 2013, 172, 1441-1449. [CrossRef] [PubMed]

12. Rey, C.; García-Hernández, I.; Concha, A.; Martínez-Camblor, P.; Botrán, M.; Medina, A.; Prieto, B.; López-Herce, J. Pro-adrenomedullin, pro-endothelin-1, procalcitonin, C-reactive protein and mortality risk in critically ill children: A prospective study. Crit. Care 2013, 17, R240. [CrossRef] [PubMed]

13. Jordan, I.; Corniero, P.; Balaguer, M.; Ortiz, J.; Vila, D.; Velasco, J.; Cambra, F.J.; Esteban, E. Adrenomedullin is a useful biomarker for the prognosis of critically ill septic children. Biomark. Med. 2014, 8, 1065-1072. [CrossRef] [PubMed]

14. Alcoba, G.; Manzano, S.; Lacroix, L.; Galetto-Lacour, A.; Gervaix, A. Proadrenomedullin and copeptin in pediatric pneumonia: A prospective diagnostic accuracy study. BMC Infect. Dis. 2015, 15, 1823. [CrossRef] [PubMed]

15. Míguez, C.; Souverbielle, C.T.; Haro, A.; Guerrero, G.; Pérez-Egido, L.; García-Gamiz, M.; Marañon, R. Evaluation of proadrenomedullin as a diagnostic or prognostic biomarker of acute appendicitis in children. Am. J. Emerg. Med. 2016, 34, 2298-2305. [CrossRef] [PubMed]

16. Esposito, S.; Di Gangi, M.; Cardinale, F.; Baraldi, E.; Corsini, I.; Da Dalt, L.; Tovo, P.A.; Correra, A.; Villani, A.; Sacco, O.; et al. Sensitivity and Specificity of Soluble Triggering Receptor Expressed on Myeloid Cells-1, Midregional Proatrial Natriuretic Peptide and Midregional Proadrenomedullin for Distinguishing Etiology and to Assess Severity in Community-Acquired Pneumonia. PLoS ONE 2016, 11, e0163262. [CrossRef] [PubMed]

17. Oikonomopoulou, N.; Míguez-Navarro, C.; Rivas-García, A.; Gamiz, M.G.; López-López, R.; Oliver-Sáez, P.; Riaño-Méndez, B.; Farfan-Orte, T.; Lobato-Salinas, Z.; Rúbies-Olives, J.; et al. Assessment of proadrenomedullin as diagnostic or prognostic biomarker of acute appendicitis in children with acute abdominal pain. Am. J. Emerg. Med. 2018, 37, 1289-1294. [CrossRef] 
18. Korkmaz, M.F.; Güzel, A.; Açıkgöz, M.; Okuyucu, A.; Alaçam, H. Reliability of Pro-adrenomedullin and Interleukin $1 \beta$ in Predicting Severity of Community-Acquired Pneumonia in Pediatric Patients. Ann. Clin. Lab. Sci. 2018, 48, 81-89.

19. Kannan, R.; Rao, S.S.; Mithra, P.; Dhanashree, B.; Baliga, S.; Bhat, K.G. Diagnostic and Prognostic Validity of Proadrenomedullin among Neonates with Sepsis in Tertiary Care Hospitals of Southern India. Int. J. Pediatr. 2018, 2018, 7908148. [CrossRef]

20. Fahmey, S.S.; Mostafa, H.; Elhafeez, N.A.; Hussain, H. Diagnostic and prognostic value of proadrenomedullin in neonatal sepsis. Korean J. Pediatr. 2018, 61, 156-159. [CrossRef]

21. Dötsch, J. Increased urinary adrenomedullin excretion in children with urinary-tract infection. Nephrol. Dial. Transplant. 1998, 13, 1686-1689. [CrossRef] [PubMed]

22. Kalman, S.; Buyan, N.; Yürekli, M.; Ozkaya, O.; Bakkaloğlu, S.; Söylemezoğlu, O. Plasma and urinary adrenomedullin levels in children with acute pyelonephritis. Nephrology 2005, 10, 487-490. [CrossRef] [PubMed]

23. Sharifian, M.; Zand, R.E.; Ahmadi, M.; Ziaee, S.A.; Mohkam, M.; Dalirani, R.D.R.; Esfandiar, N. Urinary adrenomedullin level in children with acute pyelonephritis before and after treatment. Iran. J. Kidney Dis. 2013, 7, 277-281. [PubMed]

24. Kesik, V.; Ataş, E.; Kurt, Y.G.; Aydın, F.N.; Babacan, O.; Gülgün, M.; Korkmazer, N. Adrenomedullin predicts high risk and culture positivity in children with solid tumors suffering from neutropenic fever. J. Infect. Chemother. 2016, 22, 617-621. [CrossRef] [PubMed]

25. Demirkaya, M.; Tugcu, D.; Akcay, A.; Aydoğan, G.; Akıcı, F.; Şalcığlu, Z.; Ekmekci, H.; Sevinir, B.; Ekmekci, O.B. Adrenomedullin-A New Marker in Febrile Neutropenia: Comparison with CRP and Procalcitonin. Pediatr. Hematol. Oncol. 2015, 32, 482-489. [CrossRef] [PubMed]

26. Schuetz, P.; Hausfater, P.; Amin, D.; Amin, A.; Haubitz, S.; Faessler, L.; Kutz, A.; Conca, A.; Reutlinger, B.; Canavaggio, P.; et al. Biomarkers from distinct biological pathways improve early risk stratification in medical emergency patients: The multinational, prospective, observational TRIAGE study. Crit. Care 2015, 19, 377. [CrossRef] [PubMed]

27. Liu, D.; Xie, L.; Zhao, H.; Liu, X.; Cao, J. Prognostic value of mid-regional pro-adrenomedullin (MR-proADM) in patients with community-acquired pneumonia: A systematic review and meta-analysis. BMC Infect. Dis. 2016, 16, 716. [CrossRef] [PubMed]

28. Viasus, D.; Del Rio-Pertuz, G.; Simonetti, A.F.; Garcia-Vidal, C.; Acosta-Reyes, J.; Garavito, A.; Carratalà, J. Biomarkers for predicting short-term mortality in community-acquired pneumonia: A systematic review and meta-analysis. J. Infect. 2016, 72, 273-282. [CrossRef]

29. Stalenhoef, J.E.; Van Nieuwkoop, C.; Wilson, D.C.; Van Der Starre, W.E.; Van Der Reijden, T.J.K.; Delfos, N.M.; Leyten, E.M.S.; Koster, T.; Ablij, H.C.; van Wout, J.W.V.; et al. Procalcitonin, mid-regional proadrenomedullin and C-reactive protein in predicting treatment outcome in community-acquired febrile urinary tract infection. BMC Infect. Dis. 2019, 19, 161. [CrossRef]

30. Al Shuaibi, M.; Bahu, R.R.; Chaftari, A.-M.; Al Wohoush, I.; Shomali, W.; Jiang, Y.; Debiane, L.; Raad, S.; Jabbour, J.; Al Akhrass, F.; et al. Pro-adrenomedullin as a novel biomarker for predicting infections and response to antimicrobials in febrile patients with hematologic malignancies. Clin. Infect. Dis. 2013, 56, 943-950. [CrossRef]

(C) 2019 by the authors. Licensee MDPI, Basel, Switzerland. This article is an open access article distributed under the terms and conditions of the Creative Commons Attribution (CC BY) license (http://creativecommons.org/licenses/by/4.0/). 